\title{
Delivery of a 3-month Mediterranean diet and physical activity lifestyle intervention via artificial-intelligence chatbot, can achieve behaviour change: MedLiPal pilot-study
}

\author{
Karen Murphy $^{1,2}$, Courtney Davis ${ }^{1}$, Rachel Curtis ${ }^{1}$ and Carol Maher ${ }^{1,2}$ \\ ${ }^{1}$ University of South Australia, Adelaide, Australia and \\ ${ }^{2}$ ARENA
}

\section{Abstract}

Poor diet and physical inactivity are leading modifiable causes of death and chronic disease. Robust evidence confirms that the Mediterranean diet (MedDiet) reduces mortality and cardiovascular disease risk, and promotes healthy longevity. However, MedDiet interventions are typically resource intensive, requiring regular face-to-face visits, in a one-on-one or small group setting. The rapid growth of Artificial-Intelligence (chatbot) technology has created new possibilities for low-cost, personalised health behaviour programs. We aimed to assess whether a 12-week MedDiet and physical activity intervention delivered entirely via technology (chatbot, website, wrist-worn activity tracker) could result in health behaviour change in 30 adults aged $57 \pm 8$ years. Volunteers' height, body weight, waist circumference and blood pressure were measured in clinic at baseline, week 6 and 12. Introduction of the MedDiet and physical activity recommendations were conducted through a website with short videos and written sections assisted by the chatbot. Participants were encouraged to interact with the chatbot program at least weekly for 12-weeks. The MedDiet component of the program was modelled on the MedLey trial, whilst physical activity used step count goal-setting using a Garmin Vivofit 4 wrist-worn tracker. Diet and self-reported physical activity outcomes were measured using the 14-point MedDiet questionnaire and Active Australia Survey. The study achieved 93\% retention over the 12-weeks and with excellent user engagement and satisfaction. There was a significant increase in the MedDiet score from $3 \pm 3.5$ (out of 14) at baseline, to $11 \pm 3$ at weeks 6 and 12, $(\mathrm{p}<0.001)$. Weekly moderate-to-vigorous physical activity minutes increased from $135 \pm 196$ at baseline, to $210 \pm 233$ and $300 \pm 433$ mins, at weeks 6 and 12, respectively, $(\mathrm{p}<0.001$ ). Waist circumference decreased from $99 \pm 28$ to $98 \pm 27$ and $96 \pm 25 \mathrm{~cm}$, at weeks 6 and 12, respectively, $(\mathrm{p}<0.001)$. BMI decreased from $29 \pm 10$ to $28 \pm 9$ at week 6 and $28 \pm 10$ at week $12(\mathrm{P}<0.05)$. There was no change in blood pressure. Our pilot data confirm that the chatbot together with informational website and wearable intervention approach achieved excellent user satisfaction and preliminary efficacy of behaviour change across a 12-week period. Further research employing a randomized-controlled design, and high quality outcome measures is warranted to confirm efficacy and sustainability of behaviour change. However, these early results suggest that this innovative, comprehensive chatbot-based approach may be an appealing and feasible strategy suitable for mass-dissemination to assist dietary and physical activity related behaviour, leading to a widespread reduction in risk factors for chronic disease.

\section{Conflict of Interest}

There is no conflict of interest 